\title{
Study on the Preparation of 3D Braided Carbon Fiber Reinforced Magnesium Matrix Composites
}

\section{Ping Zhang, Weinan Deng}

Guizhou University of Engineering Science, Bijie, Guizhou, 551700

\author{
Keywords: Three-Dimensional Braided Carbon Fiber; Composite; Interface; Wetting
}

\begin{abstract}
The pressure impregnation method is adopted to prepare the three-dimensional braided carbon fiber reinforced magnesium matrix composite under the protection of inert gas. The microstructure of the interface between the surface of carbon fiber and the composite was analyzed by SEM and XRD. The results show that the slight interfacial reaction between the three-dimensional braided carbon fiber with a certain thickness of SiO2 coating and the magnesium alloy matrix improves the wettability there between and effectively promotes the infiltration of magnesium alloy melt into the three-dimensional braided carbon fiber process.
\end{abstract}

\section{Introduction}

Resource conservation and environment-friendly are the major trends in the current industrial society. Research and development of lightweight, high performance, recyclable energy-saving materials are increasingly aroused widespread concern. Magnesium-based lightweight alloy with low density, high specific strength, high stiffness, recyclable and so on, is one of the hot spots in recent years. With the higher and higher requirements for high temperature performance of light metal, single alloy material can no longer meet the demand, people gradually shift their research focus to the composite material. Due to its excellent mechanical properties, low density, high thermal conductivity and low coefficient of thermal expansion, carbon fiber has become an important reinforcement in metal matrix composites [1]. Carbon fiber reinforced magnesium matrix composites not only have the characteristics of low density, high specific strength and high specific stiffness of magnesium alloys, but also can effectively improve the high temperature performance of magnesium alloys and improve the mechanical properties and thermal physical properties of the materials. Therefore, in the automotive, aerospace and other fields Industrial applications have a wide range of applications [2-3].

However, the poor wettability between carbon fiber and magnesium alloy matrix makes it difficult to form and prepare. The primary problem to be solved in the preparation of carbon fiber reinforced magnesium matrix composites is to improve the wettability between the carbon fiber and the magnesium alloy. The main technical approaches include adding alloying elements to the magnesium matrix and coating carbon fiber surfaces. However, the addition of alloying elements is usually concentrated on the fiber surface and interfacial reaction with the fiber, reducing fiber properties. Therefore, the most common and effective way to solve the wettability problem is carbon fiber surface coating. The main treatment methods are chemical vapor deposition (CVD), physical vapor deposition (PVD), electroplating, cementing, ion sputtering and sol- Gel method [4]. The sol-gel method not only has the advantages of simple process, low cost and low processing temperature, but also can coat a uniform $\mathrm{SiO} 2$ coating on the fiber surface, which is a practical treatment method.

\section{Experimental Method}

Experimental materials selected AZ31 magnesium alloy, Taiwan TCL carbon fiber TC35-12k three-dimensional carbon fiber braid. TC35-12K carbon fiber 12,000 per bunch, a diameter of about $7 \mu \mathrm{m}$. The chemicals used to prepare the sol are TEOS, A.R, A.R, and A.R.

Take a certain amount of tetraethyl orthosilicate dissolved in absolute ethanol, the proportion of 
slowly dropping quantitative deionized water, and then add the hydrolysis catalyst concentrated hydrochloric acid to adjust its $\mathrm{pH}$, while adding appropriate amount of glycerol for dry control of chemical additives (DCCA), Maintaining a constant temperature of $60^{\circ} \mathrm{C}$, under vigorous stirring, hydrolyzed tetraethylorthosilicate under acidic conditions. The sol viscosity is adjusted by the mass ratio of ethanol. The viscosity of the sol was measured with a rotary viscometer NDJ-1.

The SiO2 coating on the surface of fiber braid was prepared by sol-gel method. The process is: to glue $\rightarrow$ roughening $\rightarrow$ impregnation $\rightarrow$ drying $\rightarrow$ sintering. First carbon fiber braid immersed in acetone to glue, and then use $50 \%$ nitric acid roughened carbon fiber surface. The fiber braid is then immersed in a silica sol, immersed in a vacuum for 1 hour, and finally taken out to be dried and sintered. The morphology of the SiO2 coating on the surface of the fiber was analyzed by scanning electron microscopy. The composition of the coating on the fiber surface was analyzed by Auger electron spectroscopy. The thickness of the coating was calculated. The structure of SiO2 coating was analyzed by X-ray diffraction.

Carbon fiber reinforced magnesium matrix composites were prepared by extrusion infiltration method. Argon gas was used as the protective gas. The maximum pressure was $5 \mathrm{t}$ and the infiltration temperature was $740{ }^{\circ} \mathrm{C}$. Scanning electron microscopy and X-ray diffraction analysis of carbon fiber and magnesium matrix wetting and composite microstructure, phase composition and so on.

\section{Experimental results and discussion}

Ethyl orthosilicate does not dissolve in water to form a suspension, and the hydrolysis rate is very slow under neutral conditions. With ethanol as co-solvent, hydrochloric acid as a catalyst under conditions of agitation can accelerate the hydrolysis reaction [5]. The process usually involves two steps: one is the hydrolysis of tetraethyl orthosilicate; the other is the condensation and polycondensation of the hydroxy compound obtained after hydrolysis. The reaction of these two processes can be expressed as follows [6]

Hydrolysis: $\mathrm{Si}(\mathrm{OC} 2 \mathrm{H} 5) 4+4 \mathrm{H} 2 \mathrm{O} \rightarrow \mathrm{Si}(\mathrm{OH}) 4+4 \mathrm{C} 2 \mathrm{H} 5 \mathrm{OH}$

Polycondensation reaction: $\mathrm{Si}(\mathrm{OH}) 4+\mathrm{Si}(\mathrm{OH}) 4 \rightarrow(\mathrm{OH}) 3 \mathrm{Si}-\mathrm{O}-\mathrm{Si}$

$(\mathrm{OH}) 3 \mathrm{Si}-\mathrm{O}-\mathrm{Si}(\mathrm{OH}) 3+6 \mathrm{Si}(\mathrm{OH}) 4 \rightarrow[(\mathrm{HO}) 3 \mathrm{SiO}] 3 \mathrm{Si}-\mathrm{O}-\mathrm{Si}[\mathrm{OSi}(\mathrm{OH}) 3] 3+6 \mathrm{H} 2 \mathrm{O}$

With the hydrolysis of tetraethyl orthosilicate, the Si-O-Si bonds in the solution react with each other to form a sol. Sol particle size and degree of crosslinking can be controlled by the $\mathrm{pH}$ and the amount of water added. After the gel is formed, it is easy to cause a dry crack after the gel is dried due to the weak connection between the gel particles. In order to overcome the generation of dry crack, it is necessary to age the gel in the presence of a solvent for a period of time so that a thicker interface is formed between the gel particles and the particles, so that the gel strength gradually increases as the aging time increases, eventually enough to resist cracking due to solvent evaporation and particle shrinkage. The carbon fiber braided body was immersed in the aged sol for 1 hour under vacuum, and the particles in the sol gradually crosslinked to form a three-dimensional structural network, that is, the gelation process of the sol.

During the drying process, the solvent and the water and alcohol produced are volatilized from the system. The uneven stress caused by the drying process easily causes the gel to shrink and dry. Control of solvent, water and alcohol volatilization rate can reduce gel shrinkage and dry crack. In addition, the addition of glycerol as a dry control chemical additive (DCCA) to control the drying behavior controls the formation of size-uniformly distributed particles in the gel so that the internal stresses within the gel are uniform and not cracked after solvent evaporation. Finally, the surface of the fiber coating is completely inorganicized to form a SiO2 coating [7].

In order to make the sol have good film-forming properties, the reaction molar ratio of tetraethyl orthosilicate and $\mathrm{H} 2 \mathrm{O}$ was selected to be 1: 3 in the experiment. In this case, the formed coating is not easily peeled off due to the more complicated hydrolysis products and polymerization products. In addition, too thick $\mathrm{SiO} 2$ coating will produce serious reaction with the matrix alloy during the preparation of composite materials, resulting in a large number of reaction products, forming brittle 
layers of aggregated brittle phase and interfacial reaction products, resulting in serious fiber damage and drastic decrease of material properties [8]. In order to reduce the generation of interfacial reactants, the thickness of the fiber surface coating needs to be strictly controlled. $5 \mathrm{mPa} \cdot \mathrm{s}$. The thickness of the coating can be adjusted by the alcohol viscosity of the sol to control the viscosity of the sol prepared in this experiment maintained at about $2.5 \mathrm{mPa} \bullet \mathrm{s}$.

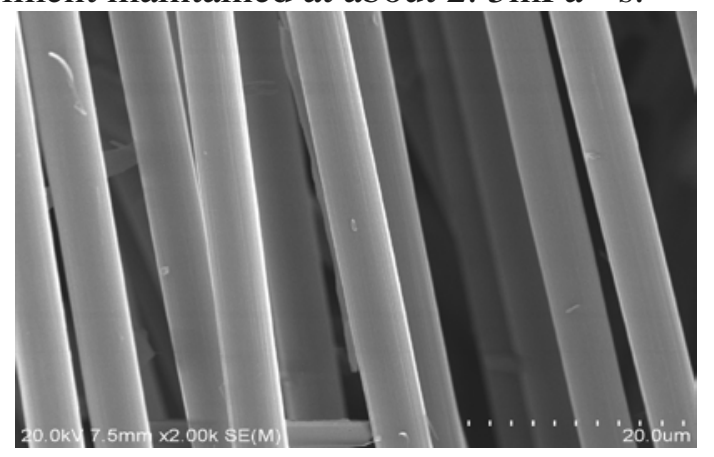

Fig. 1 SEM imagine of the carbon fibre after casting $\mathrm{SiO}_{2}$

Figure 1 shows the microstructure of carbon fiber treated by SiO2 coating. It can be seen that the fiber surface coating is transparent, uniform and bright without cracks, and the fiber filaments are separated from each other without any blocking phenomenon. Figure 2 shows the Auger electron spectroscopy analysis of carbon fiber surface coating curve, we can see that the coating contains $\mathrm{Si}$, $\mathrm{C}$, O three elements, of which carbon atoms may be Auger energy spectrum analysis caused by carbon particles Diffusion may also be caused by the diffusion of carbon in the fibers during sintering [9]. Figure 3 is the result of the Auger sputtering depth component analysis of the coating, the sputtering speed of $16 \mathrm{~nm} / \mathrm{s}$, the sputtering time of $5.8 \mathrm{~s}$ for the $\mathrm{Si}$, O element weakened to disappear, which deduced from the fiber surface $\mathrm{SiO} 2$ coating About $0.1 \mu \mathrm{m}$ or so. Figure 4 shows the results of XRD analysis. A clear peak of steamed bread only appears near the $2 \theta=25 \square$ position, indicating that the $\mathrm{SiO} 2$ coating on the carbon fiber surface has an amorphous structure.

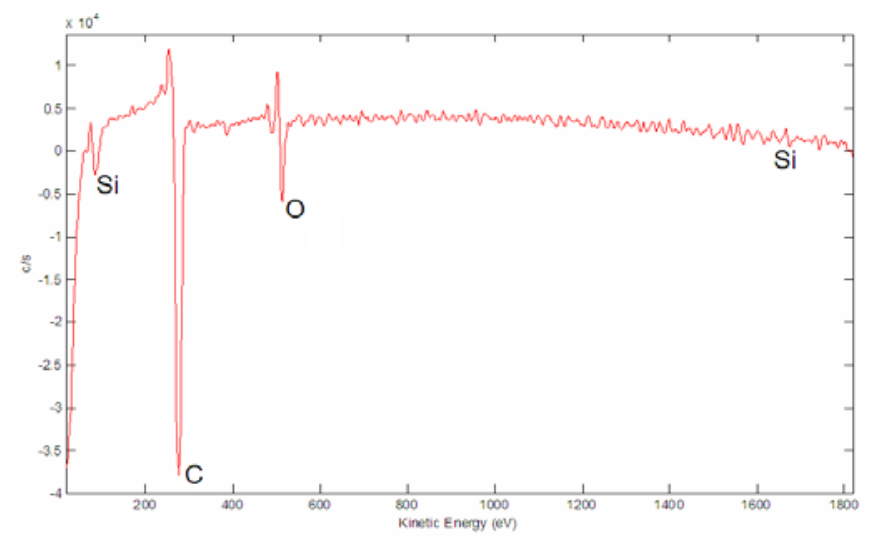

Fig. 2 Auger Energy spectrum analysis of $\mathrm{SiO}_{2}$ casted

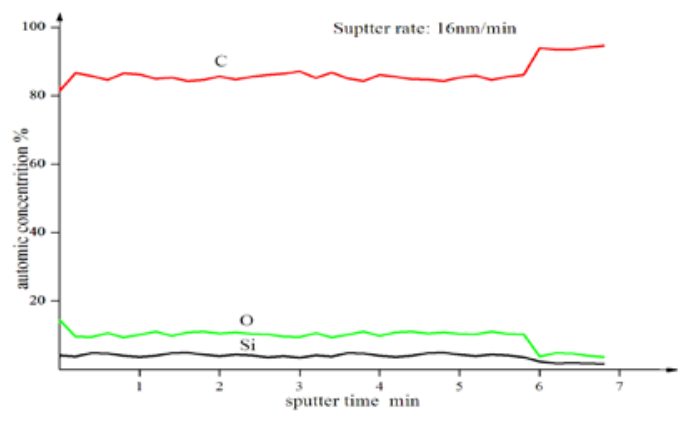

Fig. 3 distributing of composition of $\mathrm{SiO}_{2}$ costed 


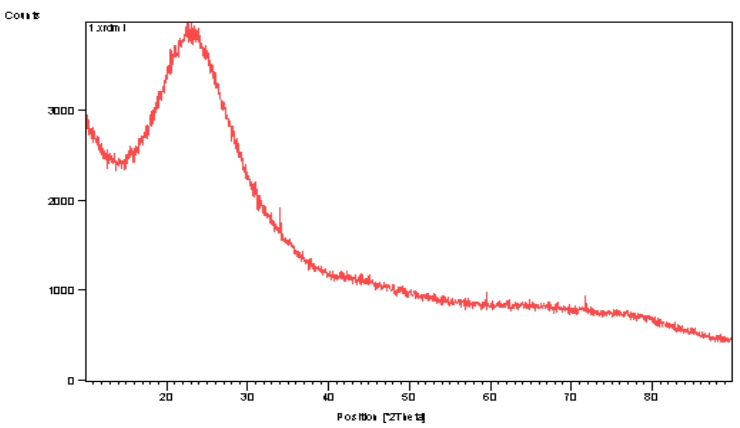

Fig. 4 X-ray diffraction patterns of $\mathrm{SiO}_{2}$ coating on the surface of carbon fibre

\section{Extrusion impregnation carbon fiber reinforced magnesium matrix composites}

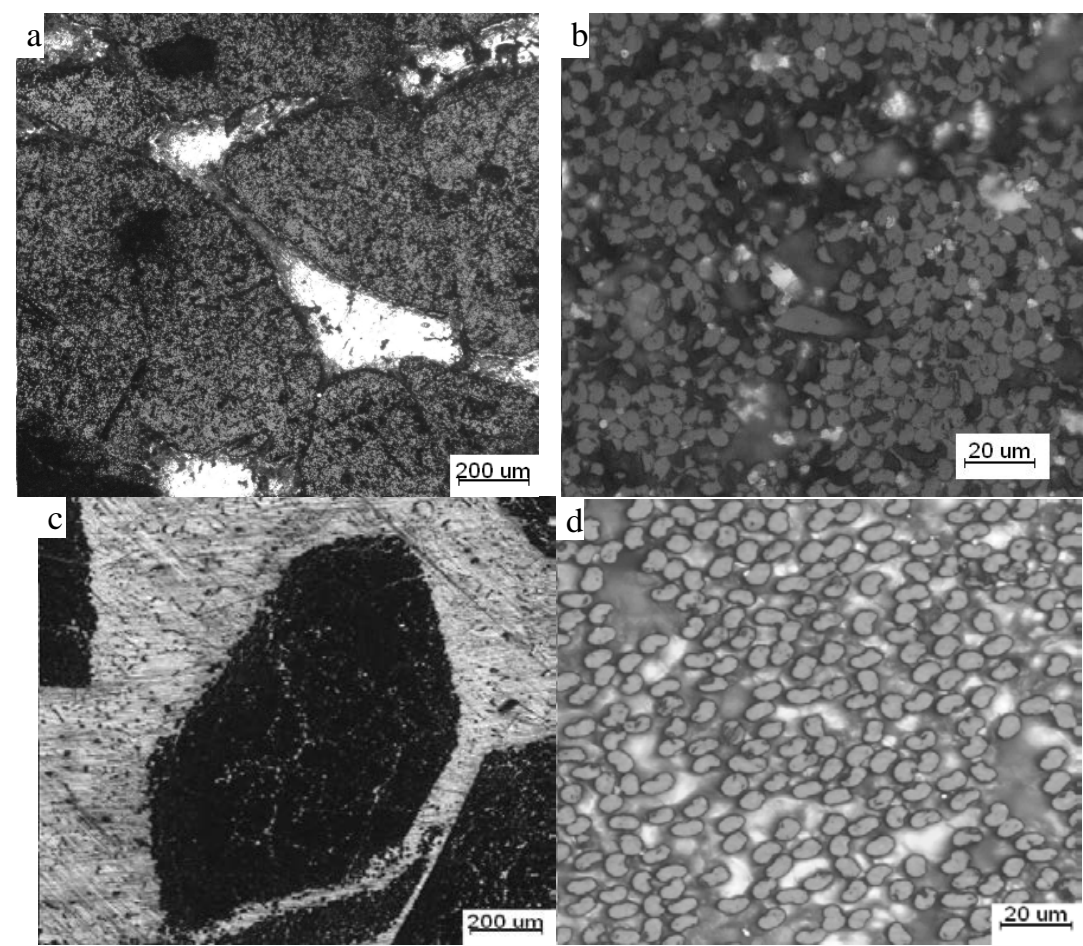

Fig. 5 Microstructure of carbon fibre reinforced Mg-based Composite

(a) and (b) for perform without $\mathrm{SiO}_{2}$ coating, (c) and (d) for perform with $\mathrm{SiO}_{2}$ coating

Figure 5 shows the microstructure of a carbon fiber reinforced magnesium matrix composite produced by extrusion infiltration under argon gas, in which the fibers in (a) and (b) are uncoated, and (c) and The fibers are treated as above. It can be seen from the figure that due to the difficulty of wetting between the magnesium alloy and the carbon fiber, the uncoated carbon fiber preform deforms only under the infiltration pressure, changing the original arrangement between the fiber bundles (Fig. 5 (a)), the carbon fibers in the fiber bundles were heavily agglomerated with little infiltration of magnesium between the filaments (Figure 5 (b)). The SiO2-treated carbon fiber reinforced magnesium-based composite material through the magnesium alloy matrix and the coating interface reaction to improve the wettability between fiber and magnesium alloy, both in the fiber bundle between the larger gap (Figure 5 (c)) and between the monofilaments in the fiber bundle (Figure 5 (d)), and the fibers were randomly distributed in the magnesium alloy matrix.

The fiber preform is prepared by using a carbon fiber three-dimensional braid. The braid has a larger gap between the fiber bundles and a smaller gap between the filaments in the braid. Therefore, the flow resistance of the metal liquid in the fiber bundles is far lower than that in the fiber bundles Flow resistance. The magnesium alloy melt infiltrates into the braid layer during infiltration and 
fills the spaces between the fiber bundles before it gradually infiltrates into the fiber bundles. Finally, the melt front contacts each other at the center of the fiber bundles and merges into Together, complete the infiltration of the fiber within the bundle. When the matrix melt is infiltrated into the fiber bundle, the external pressure is transferred by the hydrostatic pressure to the non-impregnated fibers, forcing the part of the fibers to be tightened; for fibers already covered by the melt outside the fiber bundle, the tightening force is gradually transferred to the melt through the viscous resistance between the fibers and the flowing melt, which translates into the static pressure of the melt [10].

Figure 6 shows the bonding interface between the carbon fiber and the matrix. It can be seen that the interface between the fiber and the matrix is well bonded and free of voids. Fig. 7 shows the $\mathrm{X}$-ray diffraction analysis of the prepared composite material. The presence of $\mathrm{Mg} 2 \mathrm{Si}$ and $\mathrm{MgO}$ phases in the material indicates that the molten magnesium alloy chemically reacts with the $\mathrm{SiO} 2$ coating on the surface of the carbon fiber during impregnation. By strictly controlling the thickness of the SiO2 coating during the fiber coating process, the melt undergoes only a slight chemical reaction with the $\mathrm{SiO} 2$ coating at the interface during impregnation to reduce the formation of reactants and enhance the adhesion between the fiber and the magnesium alloy substrate Between the interface bonding strength, improve the wetting ability of magnesium alloy melt and carbon fiber [11].

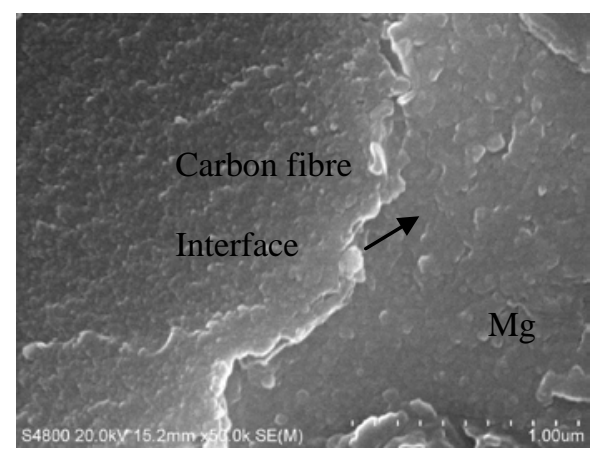

Fig.6 inteface of carbon fibre reinforced Mg-based composite

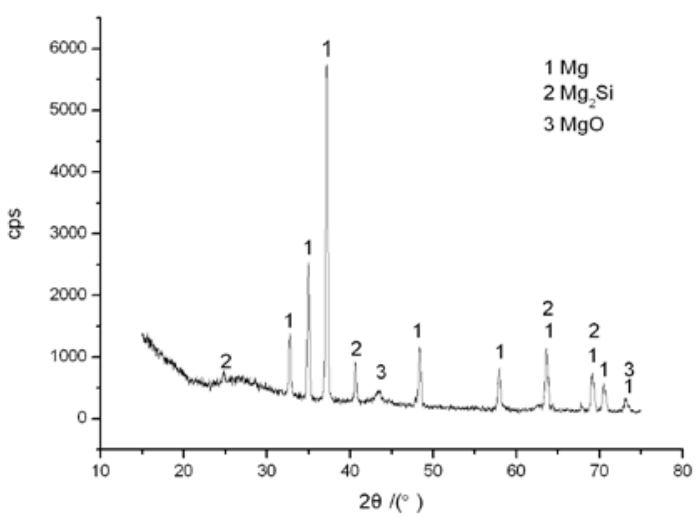

Fig .7 X-ray diffraction patterns of carbon fibre reinforced Mg-based composite

\section{Conclusion}

(1) A transparent, bright, crack-free $\mathrm{SiO} 2$ coating can be applied to the carbon fiber surface by the sol-gel method.

(2) Appropriate thickness of silica coating Carbon fiber and magnesium alloy substrate through a slight interface between the reaction to improve the wettability between the two, effectively promoted the infiltration of magnesium alloy melt in carbon fiber.

(3) Extrusion impregnation of three-dimensional braided carbon fiber reinforced magnesium matrix composite under the protection of inert gas. The material is compact and has no microscopic pores, and the interface between the carbon fiber and the magnesium matrix is well bonded. 


\section{Acknowledgements}

The Project is supported by "the Science and Technology Foundation of Gui Zhou in China” (No. Qian Ke He LKB word [2012]15)

The Project is supported by "the Guizhou Provincial Education Department Supporting Program (No. KY [2014]324)

The Project is supported by “the Science and Technology Foundation of Gui Zhou in China” (No. Qian He LH word [2014] 7518)

The Project is supported by "the Guizhou Provincial Education Department Supporting Program (No. KY [2015] 406)

The Project is supported by "the Key Laboratory Resources in Common Colleges and Universities of Guizhou Province (No. KY [2016] 010)

\section{References}

[1] Zhou Tao, Zhou Xiaoyi, A Jiancheng, Zhang Youwei, Yang Tao, Nie Zhiyun. Research progress on carbon fiber reinforced metal matrix composites [J]. Processing of Hot Processes, 2016, (No.

[2] Magna, USA, cooperates with Ford to develop carbon fiber composite engine subframe [J]. Technology Fibers and Applications, 2017, (2).

[3] Yang Minchao Design Technology and Innovation of a Heavy Duty Truck Carbon Fiber Composites Drive Shaft 201711

[4] Dong Zhijun, Li Xuanke, Yuan Guanming, Li Nan, Jiang Zhaoyang, Hu Zijun. Preparation and Application of Carbon Fiber Surface Ceramic Coatings [J]. Materials Review, 2008, (5).

[5] Zhang Rui, Qin Dandan, Wang Hailong. sol-gel methods for the preparation of SiO2 process Journal of Zhengzhou University 200627 (3) $119 \sim 122$

[6] Li Chunhua, Jiang Hong, Zhao Huifeng, Zou Shifeng. Present progress in preparation of nanoporous silica antireflection coatings by sol-gel method [J]. Glass and Enamel, 2014, (No. 4).

[7] Gao Pengzhao, Wang Hongjie, Jin Zhihao Effect of SiO2 Coating Preparation Technology on the Oxidation Resistance of Three-dimensional Carbon Fiber Braid. 200318 (4) 849 854

[8] Tao Guolin, Hu Hua Graphite (carbon) Fiber Reinforced Magnesium Matrix Composites Interface Problem Journal of Chongqing Technology and Business University 200522 (5) 497 500

[9] H. A. Katzman. Materials Science, Vol. 22, 1987, 144-148

[10] Gibson A G, Manson J A. Impregnation techmology for thermoplastic matrix composites [J]. Composites Manufacturing. 1992, 3 (4): 223-233

[11] Liu Guijun, Li Wenfang, Peng Jihua Interface reaction and thermodynamic analysis of aluminum silicate short fiber reinforced magnesium matrix composites Composite Acta Phys. Sin. 20072 (24) 8-14 